\title{
Variability in the use of CE-marked assays for in vitro diagnostics of CFTR gene mutations in European genetic testing laboratories
}

\author{
Jana Camajova $^{1}$, Sarah Berwouts ${ }^{2}$, Gert Matthijs ${ }^{2}$, Milan Macek Jr ${ }^{1}$ \\ and Elisabeth Dequeker ${ }^{\star}, 2$
}

\author{
${ }^{1}$ Department of Biology and Medical Genetics, Charles University Prague - 2nd School of Medicine and University \\ Hospital Motol, Prague, Czech Republic; ${ }^{2}$ Centre for Human Genetics, Catholic University of Leuven, Leuven, Belgium
}

DNA diagnostics of genetic diseases increasingly shifts towards utilization of commercial assays. Cystic fibrosis (CF)-related DNA diagnostics were used as a model for a pilot survey of the variability in the utilization of qualitative CE-marked in vitro diagnostic (IVD) assays and the scale of their modification by end users. A structured questionnaire, developed in the context of the EuroGentest project, was distributed within the frame of the 2005 annual CF external quality assessment (EQA) scheme. Its aim was to evaluate the variability in the use of different CE-marked IVD assays in routine CF DNA diagnostics. Survey results were analysed and sequentially discussed with respective users and/or manufacturers. In total, 125 responses from EQA scheme participants were received. Almost half of the respondents modified manufacturer-recommended protocols. They also reported sporadic and/or recurrent problems with assay performance and genotyping of particular alleles. Nonetheless, only half of the respondents performed inhouse verification before the implementation of the assay in clinical diagnostics and/or after modification of the recommended protocol. Results of this survey substantiate the importance of guidelines for proper verification of CE-marked IVD assays in DNA diagnostics, using CF as a model.

European Journal of Human Genetics (2009) 17, 537-540; doi:10.1038/ejhg.2008.209; published online 3 December 2008

Keywords: cystic fibrosis; CE-marked IVD assays; validation; verification; mutation detection; variability

\section{Introduction}

Cystic fibrosis (CF; OMIM 217900) represents one of the most commonly tested monogenic disorders within routine DNA diagnostic services in Europe and North America. CF and CFTR-related disorders are associated with a high number of different alleles in the CFTR gene (OMIM 602421). ${ }^{1-3}$ Thus, analysis of a considerable number of CFTR mutations is required to achieve a sufficiently high

*Correspondence: Professor E Dequeker, Centre for Human Genetics, Catholic University of Leuven, Campus Gasthuisberg, Herestraat 49, Leuven, B-3000, Belgium.

Tel: + 32163458 81; Fax: + 32163459 97;

E-mail: els.dequeker@med.kuleuven.be

Received 1 July 2008; revised 29 September 2008; accepted 7 October 2008; published online 3 December 2008 (ie, $>85 \%$ ) population-specific mutation detection rate. ${ }^{1}$ Utilization of complex commercial assays for routine CFTR gene testing is gradually increasing, ${ }^{2}$ as these assays provide ready-to-use solutions for the detection of up to $90 \%$ of CF-causing alleles in European-derived Caucasians. ${ }^{3}$

EuroGentest (www.eurogentest.org) is a FP6 EU Network of Excellence that primarily focuses on the harmonization and improvement of the quality of genetic services in Europe. ${ }^{4}$ Within the frame of its activities, we conducted a pilot survey whereby participants of the 2005 annual European CF external quality assessment (EQA) scheme (www.cfnetwork.be) were asked to share their experience with the use of CE-marked in vitro diagnostic (IVD) assays within routine CF DNA diagnostics. The aim of this study 
was to provide evidence supporting the necessity of verification procedures in qualitative commercial assays before their use in routine DNA diagnostics and for drafting of respective guidelines.

\section{Methods}

Variability in the use of commercial assays was evaluated by a structured questionnaire. This questionnaire was sent to all participants of the annual EQA scheme and its content was focused on the most commonly used commercial assays. These assays represent three different technical approaches to the detection of known mutations in the CFTR gene: (1) ELUCIGENE ${ }^{\mathrm{TM}}$ assays (Tepnel Diagnostics) - multiplex ARMS-based PCR followed by horizontal agarose gel electrophoresis of amplicons (versions: CF29, CF30, CF Poly-T, CF7, CF-MEP, including CF-HT, that relies on capillary electrophoresis); (2) INNO$\mathrm{LiPA}^{\mathrm{TM}}$ assays (Innogenetics) - reverse blot hybridization and post-PCR analysis based on biotin-streptavidinperoxidase sandwich hybridization and colorimetric detection with a chromogen (versions: CFTR19, CFTR17+ Tn update, CFTR $17+$ Tn, CFTR Italian Regional, CFTR12); and (3) CF Genotyping Assay ${ }^{\mathrm{TM}}$ (Abbott Diagnostics) - based on the OLA-PCR and capillary electrophoresis (versions 2 and 3). All analysed assays were CE marked. ${ }^{5}$ Results of this survey were subsequently discussed with users and/or respective manufacturer representatives.

\section{Results}

Altogether 125 responses from DNA diagnostic laboratories (further only laboratories) representing 20 countries were received. With regard to the assays tested: $17.6 \%$ of respondents used ELUCIGENE, 40.8\% used INNO-LiPA and $41.6 \%$ used the CF Genotyping Assay. From the total of 197 laboratories participating in the 2005 CF EQA scheme, 169 used one or more of these assays. As there is a similar distribution in the utilization of respective assays within the entire EQA scheme and within our survey (Figure 1), our results are representative.

However, less than half of the respondents performed inhouse verification of CE-marked IVD assays before their use in routine diagnostics (30\% for ELUCIGENE, 31\% for INNO-LiPA and $47 \%$ for CF Genotyping Assay). Moreover, owing to a variety of reasons, almost half of the respondents $(43.6 \%)$ changed the manufacturer-recommended protocols: $32 \%$ for ELUCIGENE, $41 \%$ for INNOLiPA and 58\% for CF Genotyping Assay. The most common types of modifications are listed in Figure 2.

Despite such a broad scale of modifications of recommended protocols, only $60.4 \%$ of respondents properly verified respective deviations before their implementation in routine practice. Users of CF Genotyping Assay verified

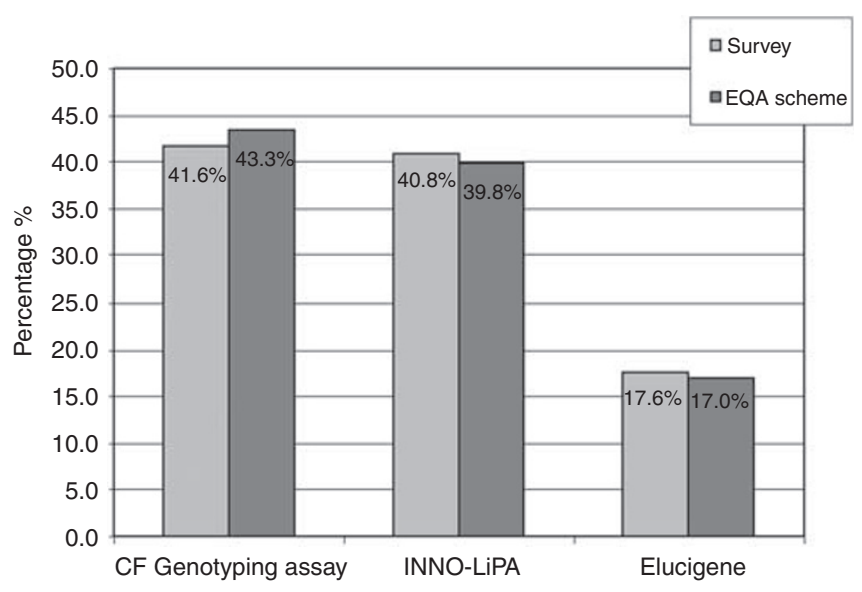

Figure 1 Proportion of the most commonly used CE-marked IVD commercial assays for CF DNA diagnostics within the survey and within the entire 2005 European CF EQA scheme.

protocol modifications in $73.3 \%$ of all cases, whereas users of ELUCIGENE and INNO-LiPA assays have done so in $55.5 \%$ and $52.4 \%$, respectively.

Almost $23 \%$ of respondents found sporadic and/or recurrent problems with correct genotyping of certain alleles and/or with the overall assay robustness (data not shown). These problems were variable and have been observed both in unmodified and/or modified assays in 16 and $84 \%$ of the cases, respectively. In addition, only 3 out of the 10 laboratories that made genotyping errors in the EQA scheme participated in this survey. Therefore, we could not assess whether there is any difference in error rate in those who modified protocols versus those who adhered to manufacturer's recommendations.

On the basis of these preliminary data, we have contacted company representatives to discuss observed problems with assay performance, discussed all issues related to the modifications of recommended protocols and suggested ways for improvement of their assays. Although this approach was highly regarded by all companies, we have not been informed whether their assays will be modified to reflect user's comments due to confidentiality measures from their side.

\section{Discussion}

In DNA diagnostics, genotyping is usually performed only once in a patient's lifetime. Therefore, it is essential to develop highly accurate testing platforms for commercial assays. This prerequisite is fulfilled by proper pre-market industrial assay development and subsequently thorough validation procedures so that manufacturers fulfil CEmarking requirements. Recently, laboratories have increasingly shifted from 'home-brew' techniques towards commercial assays and had established them as primary 


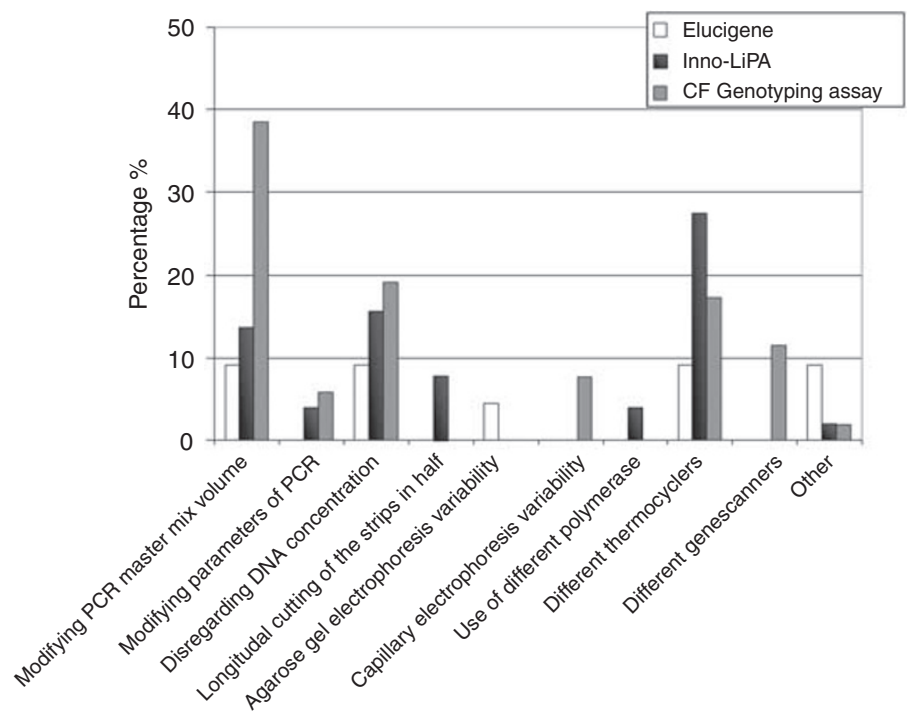

Figure 2 Examples of modifications of manufacturer-recommended protocols. Other: use of different DNA templates (eg, single-strand DNA), utilization of different software or different hybridization conditions and/or temperatures.

genotyping platforms. ${ }^{6}$ Furthermore, the growing impetus for the implementation of quality assurance and accreditation in laboratories have further accelerated this trend.

However, users should be aware of the fact that the routine implementation of commercial assays alone does not ensure high accuracy of genotyping. ${ }^{7}$ Our data gathered from the 2005 CF EQA scheme together with previous reports ${ }^{6,8}$ suggest that a considerable error rate still persists and needs to be taken into account by laboratories.

Our survey has also demonstrated that less than half of the respondents verified commercial assays before their implementation in routine diagnostic practice. Moreover, proper verification was not performed after modification(s) of manufacturer-recommended protocols. Intriguingly, the observed rate of modifications of these protocols was rather high, that is, close to $50 \%$. Although each modification should have been verified before its implementation in routine testing by laboratories, ${ }^{6,9}$ results of this survey demonstrate that only $60.4 \%$ of the respondents addressed this important issue.

In general, the rate of pre-diagnostic implementation verification of commercial assays is still insufficient and laboratories have an unsubstantiated a priori assumption that the use of a commercial assay alone assures highquality results. Moreover, laboratories frequently modified manufacturer-recommended protocols (Figure 2), but did not verify these changes. Cost-saving measures were often the main reason for modifications, for example, by cutting strips in half and correspondingly decreasing the PCR master-mix volume. In other instances, decreasing of recommended DNA template concentration was intended to avoid 'high background'. Many laboratories did not consider using recommended thermal cyclers, genetic analysers or DNA isolation techniques as essential for the overall quality of their results. Although respective modifications could be considered as neutral, advantageous or even deleterious, these should always have been verified/ validated by laboratories.

In summary, presented survey results highlight the necessity to strengthen DNA diagnostic laboratory awareness of validation/verification usefulness in all types of genetic tests, even if these comply with CE-marking requirements.

\section{Acknowledgements}

This work was supported by EuroGentest (FP6-512148), VZFNM 00064203/6112/ and EU CF Network. We are grateful to all laboratories that took part in the survey, including Drs A Corveleyn and F Le Calvez, who contributed to the development of the questionnaire. We appreciate the encouraging role of Professor J-J Cassiman. Input from Abbott Molecular, Innogenetics and Tepnel Diagnostics was helpful.

\section{References}

1 Dequeker E, Cuppens H, Dodge $\mathrm{H}$ et al: Recommendations for quality improvement in genetic testing for cystic fibrosis. European Concerted Action on Cystic Fibrosis. Eur J Hum Genet 2000; (Suppl 2): S2-S24.

2 Dequeker E, Cassiman JJ: Genetic testing and quality control in diagnostic laboratories. Nat Genet 2000; 25: 259-260. 
3 Girodon-Boulandet E, Cazeneuve C, Goossens M: Screening Practices for Mutations in the CFTR Gene ABCC7. Hum Mutat 2000; 15: 135-149.

4 Cassiman JJ: Research network: EuroGentest - a European Network of Excellence aimed at harmonizing genetic testing services. Eur J Hum Genet 2005; 13: 1103-1105.

5 Directive 98/79/EC of the European Parliament and of the Council. The European Parliament and the Council of the European Union 1998, http://www.ivd-ce.com/ivd.pdf (Accessed October 2007).
6 Dequeker E, Ramsden S, Grody WW, Stenyel TT, Barton DE: Quality control in molecular genetic testing. Nat Rev Genet 2001; 2: 717-723.

7 Prence E: A practical guide for the validation of genetic tests. Genet Test 1999; 3: 201-205.

8 Ramsden SC, Deans Z, Robinson DO et al: Monitoring standards for molecular genetic testing in the United Kingdom, the Netherlands, and Ireland. Genet Test 2006; 10: 147-156.

9 OECD Guidelines for Quality Assurance in Molecular Genetic Testing, OECD 2007, http://www.cfnetwork.be/doc/QAGuidelineseng. pdf (Accessed May 2007). 